\title{
High Resolution TEM Observation of Nanocrystalline Silicon Fabricated by High Pressure Torsion (HPT)
}

Yuta Fukushima ${ }^{1,2}$, Kaveh Edalati ${ }^{1,3}$, Yoshifumi Ikoma ${ }^{1}$, Zenji Horita ${ }^{1,3}$, and David J. Smith ${ }^{2}$

${ }^{1 .}$ Department of Materials Science \& Engineering, Kyushu University, Fukuoka 819-0395, Japan

2. Department of Physics, Arizona State University, Tempe, Arizona 85287, USA

${ }^{3 .}$ WPI, International Institute for Carbon-Neutral Energy Research (WPI-I2CNER), Kyushu University, Fukuoka 819-0395, Japan

Silicon has 12 different crystal structures under different pressure [1]. Si has the cubic diamond (cd) structure ( $\mathrm{Si}$-I) under pressure up to $\sim 11.7 \mathrm{GPa}$. Si-I transforms to the metallic $\beta$-tin structure (Si-II) above $\sim 11.7 \mathrm{GPa}$. Si-II transforms to the R8 structure (Si-XII) at $\sim 8 \mathrm{GPa}$ to $\mathrm{Si}$-I when pressure is released slowly. Si-XII may transform subsequently to the BC8 structure ( $\mathrm{Si}$-III) upon releasing the pressure. Si-XII and $\mathrm{Si}$-III transform to the hexagonal diamond (hd) phase ( $\mathrm{Si}-\mathrm{IV}$ ) by subsequent annealing at moderate temperature [1,2]. Si-IV also can transforms to Si-I by prolonged annealing. In this study, the effect of severe plastic deformation (SPD) on pressure-induced phase transformations is investigated using the high-pressure torsion (HPT) method, which is originally used to improve the mechanical properties of alloys by producing ultrafine-grained (UFG) structures [3].

The HPT facility used in this study consisted of two anvils with a hole $(5 \mathrm{~mm}$ diameter and $0.25 \mathrm{~mm}$ thickness) on the surface of each anvil. Two Si (100) wafers were placed on the hole of the lower anvil, and then slowly compressed under a pressure of $24 \mathrm{GPa}$. After compression, the lower anvil was rotated with respect to the upper anvil for 20 turns using a rotation speed of $1 \mathrm{rpm}$ at room temperature. After HPT processing, some samples were annealed at $873 \mathrm{~K}$ for $1,2,6,12$ hours under $\mathrm{N}_{2}$ flow $(0.1 \mathrm{~L} / \mathrm{m})$. For transmission electron microscopy (TEM), samples were prepared by crushing in isopropanol and deposition onto holey carbon films. Nanostructures were examined by phase-contrast high-resolution TEM using a JEOL JEM-4000EX operated at $400 \mathrm{keV}$ and a FEI-Philips CM200-FEG operated at 200 $\mathrm{keV}$. High-resolution images were analyzed using Gatan DigitalMicrograph software.

Examination of TEM images indicates that multiple metastable high-pressure phases are formed by HPT processing. Figure 1(a) shows the presence of Si-XII phase with orientations of [112] and [0-1-1]. Other diffraction spots in the diffractogram of Fig. 1(a) correspond to Si-I phase. Figure 1(b) suggests the coexistence of Si-I and Si-IV with orientations of [110] and [100], respectively. The presence of many spots in the diffractogram of Fig. 1(a) and (b) indicates the existence of small nanograins with sizes in the nanometer scale. The grain boundaries are shown more clearly in Fig. 1(b). After annealing, Si-I is the only detected phase, consistent with an earlier report [4]. The grain sizes remain in the nanometer range after annealing and many dislocations, as shown in Fig. 2(a), and many nanotwins with a typical lamella width in the range of 2-3 \{111\} atomic fringes, as shown in Fig. 2(b), are formed. These lattice defects also form after HPT processing, but their fraction increases after annealing [5].

References:

[1] B. D. Malone, J. D. Sau, and M. L. Cohen, Phys. Rev. B78, 035210 (2008).

[2] M. Cohen and B. Malone, J. Appl. Phys. 109, 102402 (2011).

[3] R. Z. Valiev, A.V. Korznikov, R. R. Mulyukov, Mater. Sci. Eng. A168, 99 (1993).

[4] Y. Ikoma, et al, J. Mater. Sci. 49, 19 (2014). 
[5] This work was supported in part by a Grant-in-Aid for Scientific Research from the MEXT Japan, in Innovative Areas "Bulk Nanostructured Metals" (Nos. 22102004, 25102708). The authors gratefully acknowledge use of facilities in the John M. Cowley Center for High Resolution Microscopy at Arizona State University.

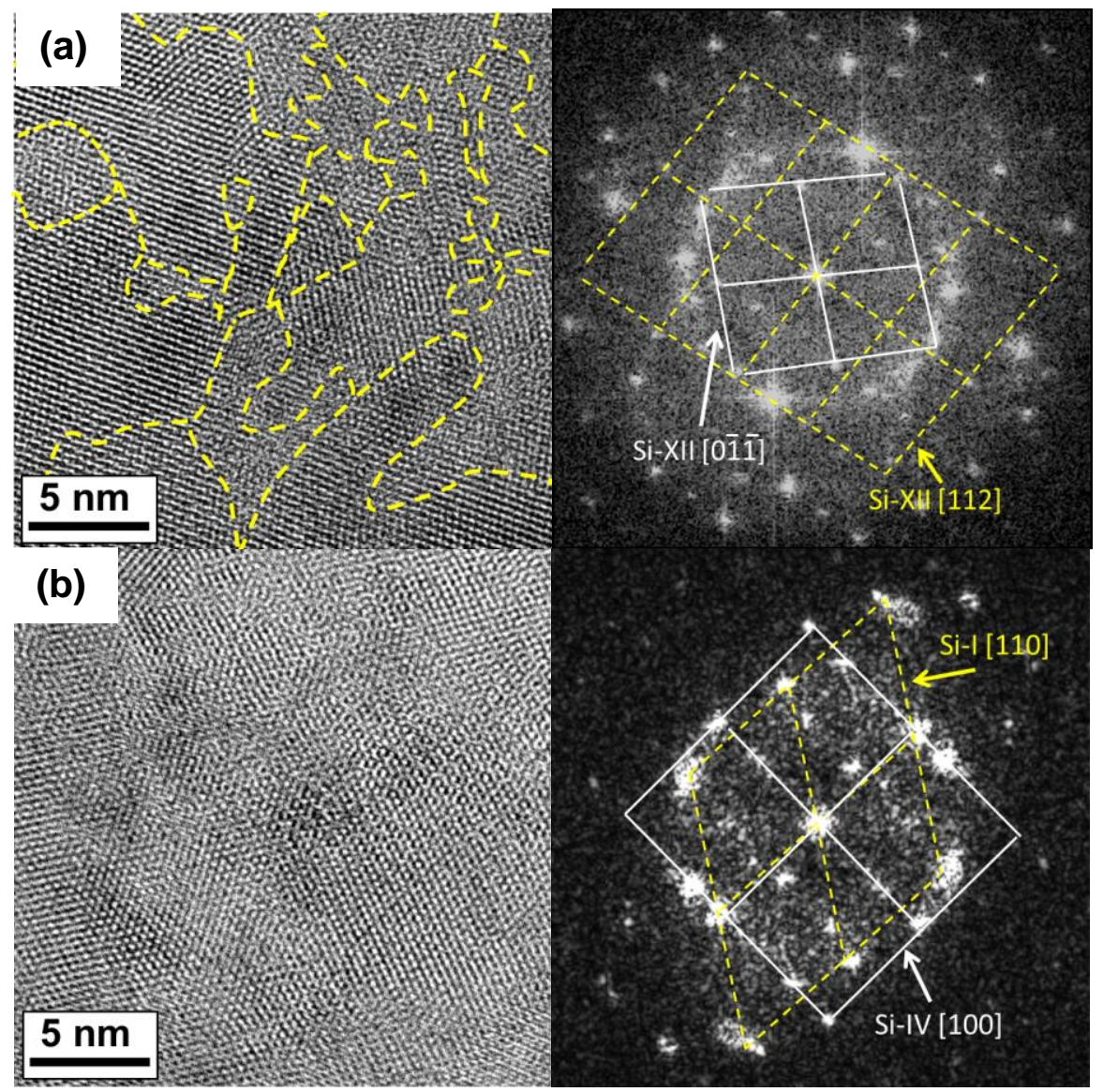

Figure 1. TEM lattice images (left), and corresponding diffractograms (right), for Si after HPT processing. Formation of metastable Si-XII and Si-IV are shown in (a) and (b), respectively.

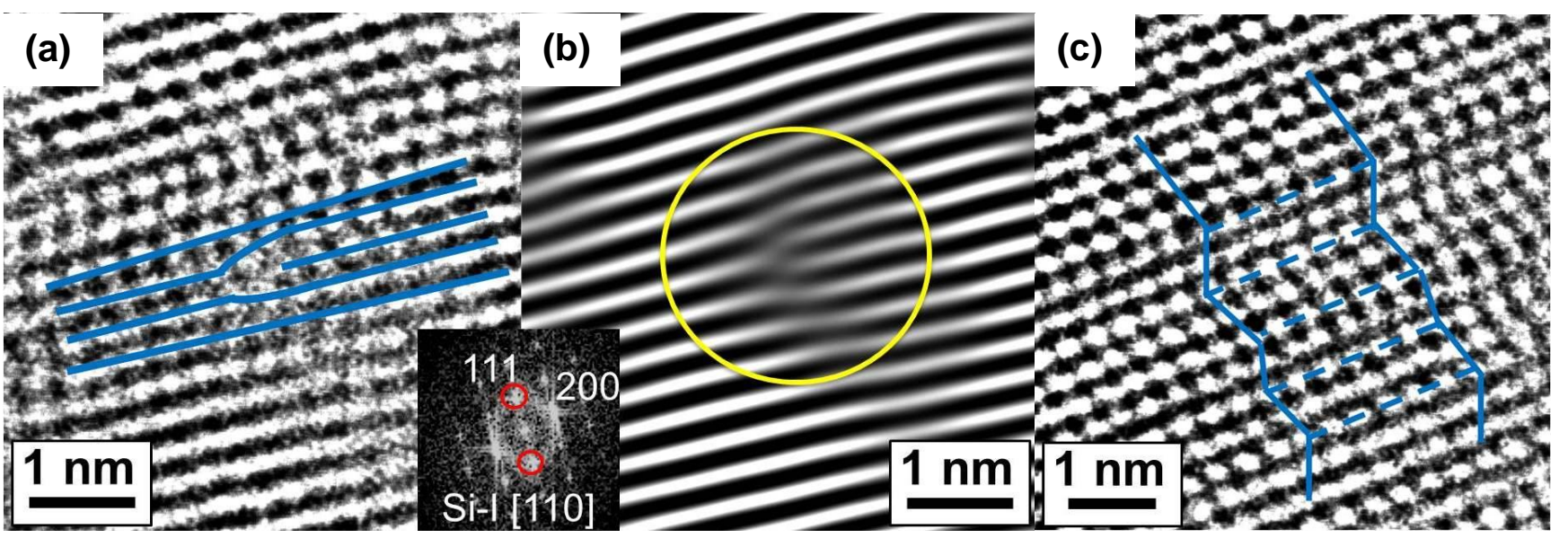

Figure 2. (a) Lattice image of $\{111\}$ dislocation and (b) corresponding inverse FFT constructed using [111] diffraction spots. (c) Lattice image of Si nanotwins present after annealing. 